\title{
THE EFFECTS OF RADIO-FREQUENCY RADIATION (RFR) EXPOSURE ON THE ANALGESIC EFFICACY OF MORPHINE IN HEALTHY RATS AND RATS WITH INFLAMMATION
}

\section{PAWEŁ BODERA ${ }^{1}$, BOŻENA ANTKOWIAK ${ }^{2}$, MAŁGORZATA PALUCH ${ }^{3}$, BAHRIYE SIRAV $^{4}$, ANDRZEJ K. SIWICKI $^{5}$, and WANDA STANKIEWICZ ${ }^{1}$}

\author{
${ }^{1}$ Military Institute of Hygiene and Epidemiology, Warsaw, Poland \\ Department of Microwave Safety \\ ${ }^{2}$ Military Institute of Hygiene and Epidemiology, Warsaw, Poland \\ Department of Pharmacology and Toxicology \\ ${ }^{3}$ Eppendorf Poland Ltd., Warsaw, Poland \\ ${ }^{4}$ Gazi University, Beşevler, Ankara, Turkey \\ Faculty of Medicine, Department of Biophysics \\ ${ }^{5}$ University of Warmia and Mazury, Olsztyn, Poland \\ Department of Microbiology and Clinical Immunology
}

\begin{abstract}
Objectives: The aim of this study, conducted at the Military Institute of Hygiene and Epidemiology in Warsaw in 2017, was to evaluate the effects of a single (15 $\mathrm{min})$ and repeated (5 times for $15 \mathrm{~min}$ ) radio-frequency radiation (RFR) exposure of $1800 \mathrm{MHz}$ frequency on the analgesic efficacy of morphine in healthy rats and rats with complete Freund's adjuvant (CFA) induced inflammation. Material and Methods: Rats were injected intraperitoneally with morphine (MF) in the dose of $8 \mathrm{mg} / \mathrm{kg}$ or drug vehicle $15 \mathrm{~min}$ before RFR exposure. The authors used the plantar analgesia meter and the radiant heat paw-withdrawal test to assess the pain threshold. Results: A single RFR exposure slightly influenced paw withdrawal latency (PWL) in healthy rats in the single exposure baseline group, and influenced PWL, 30 and 60 min after morphine or vehicle injection, in the repeated exposure group. There were differences between the sham-exposed groups (vehicle), 30, 60 and 90 min after injection, both in the single and repeated RFR-exposure groups. The antinociceptive effect of morphine in healthy rats was slightly decreased by RFR exposure at 60 and 90 min, both in the single and repeated exposure groups. The PWL was slightly decreased, both in the single and repeated exposure groups with inflammation (CFA and CFA/MF), at 30, 60 and $90 \mathrm{~min}$, and PWL was increased in the sham-exposed groups (CFA and CFA/MF), both in the single and repeated exposure groups, at 30, 60 and $90 \mathrm{~min}$. The antinociceptive effect of morphine in healthy rats was significantly increased by RFR exposure at $30 \mathrm{~min}$ after drug injection in the single exposure group, and increased at 30 and $60 \mathrm{~min}$ in the repeated exposure group. Conclusions: The authors observed a minor influence of RFR exposure on the antinociceptive effects of morphine in healthy rats after repeated exposures and a statistically significant influence of repeated exposure on morphine mediated antinociceptive effects in the inflammation group. Int J Occup Med Environ Health. 2019;32(4):465-74
\end{abstract}

Key words:

inflammation, rats, morphine, pain perception, radio-frequency radiation, paw withdrawal latency

Received: October 12, 2017. Accepted: March 11, 2019.

Corresponding author: Wanda Stankiewicz, Military Institute of Hygiene and Epidemiology, Department of Microwave Safety, Kozielska 4, 01-163 Warsaw, Poland (e-mail: wanda.stankiewicz@gmail.com). 


\section{INTRODUCTION}

Radio-frequency radiation (RFR) is a universal and ubiquitous factor, widely present in the environment, which may interact with endogenous neurochemistry and pharmacological treatments. The rapid growth of communication systems and the large number of time-varying patterns generated directly by electronic devices, such as computers, Wi-Fi systems, and light sources, may have a major influence on living systems. An extraordinary number of different RFR signals, generated by carrier frequencies within the $\mathrm{GHz}$ range, overlap within this range; they are propagated simultaneously and may interact with neurochemical loops in the brain, consisting of receptors and neurochemical messengers, and may ultimately influence the stability of the blood-brain barrier [1-4].

Numerous data have shown that electromagnetic fields with different values can have an influence on releasing or inhibiting endogenous opioids or enhancing/decreasing the activity of opioid signaling pathways. Zecca et al. [5] found that exposure for 8 months to electromagnetic fields (RFR) of 2 different field strength combinations, i.e., $5 \mu \mathrm{T}$, $1 \mathrm{kV} / \mathrm{m}$ and $100 \mu \mathrm{T}, 5 \mathrm{kV} / \mathrm{m}$, affected the opioid system, which was involved in key changes in the frontal cortex, parietal cortex, and hippocampus, and connected with an increase in the norepinephrine levels in the pineal gland in rats. They suggested that RFR might cause alteration of several brain functions.

Bao et al. [6] suggested that repeated magnetic field exposures might have an influence on the analgesic effect. They reported that 4-day exposure increased the levels of $\beta$-endorphin and substance $P$ in the hypothalamus of rats. In addition, a morphine-induced decrease in dopamine D2 receptor (D2R) density in the rat dorsal hippocampus, after exposure to RFR, was reported by Wang et al. [7]. The results showed that the density of D2Rs in shamexposed morphine-treated rats on the first and third day of morphine withdrawal was significantly lower than in the saline control group. The results suggest that dorsal hippocampal D2Rs are potentiated by RFR pre-exposure during morphine treatment and are sensitive to morphine withdrawal.

This research was designed to evaluate the possible influence on pain perception, in healthy rats and rats with inflammation, of exposure to $1800 \mathrm{MHz}$ RFR.

\section{MATERIAL AND METHODS \\ Animals}

Male Wistar rats weighing 220-250 g, purchased from the Center of Experimental Medicine (Medical University of Białystok, Poland), were used in this study and were exposed to a standard 12:12 h light/dark cycle with water and food made available ad libitum. Approximately $1 \mathrm{~h}$ before the experiments began, the animals were transported to the laboratory and all behavioral testing was performed between 9:00 a.m. and 4:00 p.m. Each animal was only tested once. All the animal care and handling procedures were in accordance with the guidelines of the International Association for the Study of Pain (IASP) on the use of animals in pain research.

\section{Drugs and chemical compounds}

A solution of morphine sulfate (MF) $(10 \mathrm{mg} / \mathrm{ml}$, morphine sulfate WZF, Polfa Warszawa, Poland) was prepared in saline. Complete Freund's adjuvant (CFA) - heat killed Mycobacterium tuberculosis suspended in paraffin oil, $1 \mathrm{mg} / \mathrm{ml}$ - was purchased from Sigma-Aldrich.

\section{Complete Freund's adjuvant}

Persistent pain and localized inflammation was induced in the footpad of the rats by the injection of complete Freund's adjuvant (composed of inactivated and dried Mycobacterium and adjuvant) [8]. A Mycobacterium butyricum oil suspension was initially used to induce adjuvant arthritis and persistent pain by inoculation of the tail base of the rat [9]. After a CFA injection into the footpad, cutaneous inflammation appears in minutes to hours, peaking 
within 5-8 h [10]. A dose-dependent inflammatory response in the injected hind paw appears after the application of Mycobacterium butyricum suspended in oil/saline (1:1) with significant edema and thermal hyperalgesia. The edema peaks around $24 \mathrm{~h}$ after the injection without any sign of an immune response or systemic disease. The physiological and biochemical effects of CFA are only observed in the affected limb [10]. No significant alterations were observed in an open field locomotion test in rats with CFA-induced inflammation. In addition, minimum reductions in weight were observed and the animals showed normal grooming behavior.

\section{Equipment}

A plantar analgesia meter, Model 336 Analgesia Meter (IITC, Inc./Life Science Instruments, Woodland Hills, CA, USA), was used during the radiant-heat paw-withdrawal test to assess thermal sensitivity [11]. To measure the nociceptive response to a noxious heat stimulus, each animal was placed in a Plexiglas chamber on a glass plate located above a light box. The noxious stimulus consisted of a high-intensity beam from a projector lamp bulb located below the glass floor, which was aimed at the midhind paw, and the latency in seconds to paw withdrawal was recorded.

The animal was able to move freely on the glass surface and when the rat was stationary, an infrared source was focused under the animal and heat applied to the plantar surface of the foot. A photosensor was used to detect paw movement, and the latency from heat onset to paw withdrawal was recorded. To avoid sensitization of the response, each animal was tested in sequential trials at approximately 5-min intervals.

Radiant heat from the Model 336 Analgesia Meter was applied by pointing a beam of light at the middle of the plantar surface of each hind paw. When the animal lifted its foot, the light beam was turned off. The length of time between the start of the light beam and pain response was defined as the paw withdrawal latency (PWL). Each trial was repeated twice, at 5-min intervals, for each paw. A cutoff time of $20 \mathrm{~s}$ was used to avoid paw tissue damage.

This method has some advantages as compared to the tail-flick assay because the plantar surface on the foot is sensitive sensory skin, which is comparable to other mammalian skin surfaces. Furthermore, the stress level of the test animals is decreased because they are not manually restrained as in the tail-flick assay or in the immersion test. The glass was heated to $30^{\circ} \mathrm{C}$ to minimize variations in the baseline temperature of the skin, to prevent paw cooling and to ensure that the tested paw is in contact with the glass [12].

\section{Experimental procedure}

Eight subgroups of male Wistar rats were randomly assigned ( $N=64, N=8$ per group ). The groups of healthy rats and rats with inflammation, in which the vehicle was injected, were divided in a similar way (Table 1). In order to assess the influence of RFR exposure on the nociceptive threshold and MF analgesia, both in normal and inflammatory state conditions, 15 min before RFR exposure, rats were intraperitoneally injected with MF in the

Table 1. Distribution of the groups of rats, based on their condition, in the study of the influence of radio-frequency radiation (RFR) exposure on the analgesic efficacy of morphine, conducted at the Military Institute of Hygiene and Epidemiology in Warsaw in 2017

\begin{tabular}{lcc}
\hline \multirow{2}{*}{ Group of rats } & \multicolumn{2}{c}{ Group } \\
\cline { 2 - 3 } & sham-exposed & RFR-exposed \\
\hline Healthy & & \\
treated & $\mathrm{MF}$ & $\mathrm{MF} / \mathrm{RFR}$ \\
untreated & control & $\mathrm{RFR}$ \\
Inflammated & & \\
treated & $\mathrm{CFA} / \mathrm{MF}$ & $\mathrm{CFA} / \mathrm{MF} / \mathrm{RFR}$ \\
untreated & $\mathrm{CFA}$ & $\mathrm{CFA} / \mathrm{RFR}$ \\
\hline
\end{tabular}

CFA - complete Freund's adjuvant; MF - morphine; RFR - radio-frequency radiation. 
dose of $8 \mathrm{mg} / \mathrm{kg}$ or vehicle in the volume of $1 \mathrm{ml} / \mathrm{kg}$. The rats that were not injected with MF or the vehicle, and that were not exposed to RFR, formed the control group.

\section{Electromagnetic field exposure system}

The electromagnetic field (EMF) exposure system consisted of:

- Rohde \& Schwarz SMT06 Signal Generator (5 kHz$6 \mathrm{GHz}$ ),

- TMD PTC6341 Amplifier (0.8-2 GHz, 250 W),

- Rohde \& Schwarz NRT-Z44 Directional Power Sensor (0.2-4 GHz, 0.003-120 W),

- EMCO 3115 Waveguide Horn Antenna (1-18 GHz).

The exposure containers with 2 rats each and verticallypolarized antenna were placed inside the anechoic chamber. Four exposure containers per group were used.

The rats, which had limited freedom of movement, were exposed in pairs inside the double box transparent plastic exposure container. The outer dimensions of the container were $218 \mathrm{~mm}$ in length and width, and the container height was $134 \mathrm{~mm}$. The container walls were $5 \mathrm{~mm}$ thick. The rats in other groups were also placed in the exposure container, but they were not exposed to RFR stimulation. Persistent inflammation was induced by an injection of CFA into the plantar surface of the left hind paw (in a volume of $0.1 \mathrm{ml}$ ) $24 \mathrm{~h}$ before RFR exposure and drug application, and the assessment of thermal nociception was performed using the plantar test according to the Hargreaves method [11]. The 1st measurement of PWL was recorded $30 \mathrm{~min}$ before morphine or vehicle administration in the 4 groups of healthy rats and the 4 groups of rats with inflammation (control, RFR-exposed, MF and MF/RFR). This was adopted as the baseline for the further PWL measurements performed after 30, 60 and 90 min (Figure 1).

The same number of rats were either RFR-exposed or sham-exposed (baseline; with no voltage applied to the field generator) and sham-exposed treated with morphine. Animals were exposed to RFR for 15 min for a single ex-

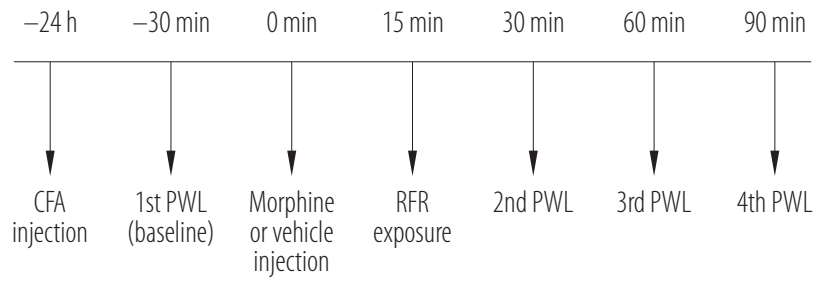

CFA - complete Freund's adjuvant; PWL - paw withdrawal latency.

Figure 1. Schematic representation of the experiment timeline the influence of the $1800 \mathrm{MHz}$ radio-frequency radiation (RFR) exposure on the analgesic efficacy of morphine in healthy rats and rats with complete Freund's adjuvant (CFA) induced inflammation - the study conducted at Military Institute of Hygiene and Epidemiology in Warsaw in 2017

posure or repeated exposures, once a day, for 5 consecutive days. It was attempted to establish a mobile phone exposure-like situation.

The animals in each group were continuously exposed to the far-field range of an antenna at $1800 \mathrm{MHz}$, with an electric field of $20 \mathrm{~V} / \mathrm{m}$ and an magnetic field of $0.05 \mathrm{~A} / \mathrm{m}$. The propagation vector of the incident wave was parallel to the long axis of the animal's body. The PWL to a thermal stimulus was then measured 30, 60 and 90 min after drug or vehicle injections.

\section{Specific absorption rate (SAR)}

The specific absorption rate (SAR) was calculated using a numerical model for rats exposed to a plane wave polarized RFR. The authors used a finite-difference-time-domain (FDTD) simulation with a simplified homogeneous rat model. The 3-D anatomical model used in FDTD numerical calculations was acquired from Duke University and included a realistic description of the organs and skeletal system of the rat [13].

In order to use precise shapes in the rat models, images of the animals were taken during the exposure, in addition to MRI scans of the rat body, and biometric data of the exposed animals were used to add missing anatomical features, such as the tail, to the model. Non-uniform rational basis spline functions (B-splines) obtained from 
the MRI scans were used to create a high-resolution anatomical model of the Sprague-Dawley rat (voxel dimensions $0.39 \times 0.39 \times 0.41 \mathrm{~mm})$ and to predict normalized SAR values $\left(\mathrm{W} / \mathrm{kg}\right.$ per $\left.\mathrm{mW} / \mathrm{cm}^{2}\right)$. This anatomical model was developed by segmentation and 3-D reconstruction of medical images (81 images, $3 \mathrm{~mm}$ thick) that were obtained from the Brooks Air Force Base [14].

Each rat model had an assumed average rat body density of $1.06 \mathrm{~g} / \mathrm{cm}^{3}$ and a body weight of approximately $231 \mathrm{~g}$. The average conductivity and permittivity of the rat body was equal to that of the human body which is equivalent to two-thirds that of muscle tissue. In order to achieve the optimal exposure conditions, the following 3 exposure scenarios were analyzed:

- when the rats are positioned on the box floor and the wave vector $(\mathrm{k})$ is parallel to the long axis of the animal,

- when both rats are positioned on the box floor opposite the antenna,

- when both rats are positioned as if climbing on the front wall of the box.

The first scenario revealed the minimum possible value of the SAR, and in the third scenario the maximum SAR value was predicted to occur with the SAR values calculated, ranging $0.024-0.028 \mathrm{~W} / \mathrm{kg}$.

In order to validate the SAR calculations, the rat model dimensions were increased to a volume of $314 \mathrm{~cm}^{3}$, and the plane wave condition, in this case, had the electric field vector parallel to the long axis of the model. The root mean square (RMS) value of the electric field strength calculated was $61.4 \mathrm{~V} / \mathrm{m}$, which was equal to $1 \mathrm{~mW} / \mathrm{cm}^{2}$ at the plane wave condition (at a frequency of $1800 \mathrm{MHz}$ ). The results of this calculation are consistent with the whole body SAR value that was calculated in the medium-sized rat model, whereas the volume of the presented model was $320 \mathrm{~cm}^{3}$ [15]. The front wall of the exposure box in the anechoic chamber was $1.2 \mathrm{~m}$ from the antenna aperture. The generator produced a carrier wave at a frequency of $1800 \mathrm{MHz}$ with the pulse modulation at a duration of $0.577 \mathrm{~ms}$, which repeated every $1.14 \mathrm{~ms}$. The basis of the GSM utilizes time division multiple access (TDMA) and frequency division multiple access (FDMA). Therefore, this involves the division of the (max) $25 \mathrm{MHz}$ bandwidth by frequency into 124 carrier frequencies spaced $200 \mathrm{kHz}$ apart.

The assumption was made that 1 carrier frequency is divided in time using a TDMA scheme, which is assigned to the base station. The fundamental unit of time in this scheme, equal to approximately $0.577 \mathrm{~ms}$, and lasting for $15 / 26 \mathrm{~ms}$, was defined as a burst period. The basic unit of a logical channel was formed from 8 burst periods grouped into a TDMA frame (120/26 ms or approximately $4.615 \mathrm{~ms}$ ). The strength of the electric field is determined by the distance from the base station to the subscriber that occupies a particular time slot, which is regulated automatically in each unit of time. The simplified base station signal used in this study was replaced with a $0.577 \mathrm{~ms}$ pulse train containing $50 \%$ of the duty cycle. This was applicable to the conditions when the subscribers that remained far-distant and near to a base station antenna were alternately assigned to the time slots to the antenna aperture generated an electric field RMS value which totaled at $20 \mathrm{~V} / \mathrm{m}$ when it was located $1.2 \mathrm{~m}$ from the center of the exposure box. The results of the above calculation were verified by means of a Wandel \& Goltermann EMR-200 electric field meter with probe No. 9 (a measurement range of $0.01-18 \mathrm{GHz}$, $0.5-1000 \mathrm{~V} / \mathrm{m})$. The calibration of this meter was performed by the Laboratory of Electromagnetic Field Standards and Metrology at the Wrocław University of Science and Technology, Poland.

\section{Ethics}

The experimental procedures were carried out in accordance with the European Community (Directive 2010/63/ EU for animal experiments [16]) and the National Institutes of Health guidelines for the care and use of laboratory animals [17]. All experimental work was approved by the local ethics committee for animal experimentation. 


\section{Statistical analysis}

For statistical calculations, the authors used the 2-way analysis of variance (ANOVA). The significance of the differences observed between the groups was verified with the Dunnett test. A value of $p<0.05$ was considered statistically significant. Statistical analysis was performed using GraphPad Prism version 6.00 for Windows (GraphPad Software, La Jolla, CA, USA).

\section{RESULTS}

\section{Healthy rats}

Exposure to a frequency of $1800 \mathrm{MHz}$ with an electric field intensity of $20 \mathrm{~V} / \mathrm{m}$, with both a single and repeated RFR exposure, did not markedly influence the nociceptive threshold to a thermal stimulus (PWL) in healthy rats (Figures 2 and 3). The PWL in the healthy rats group, from

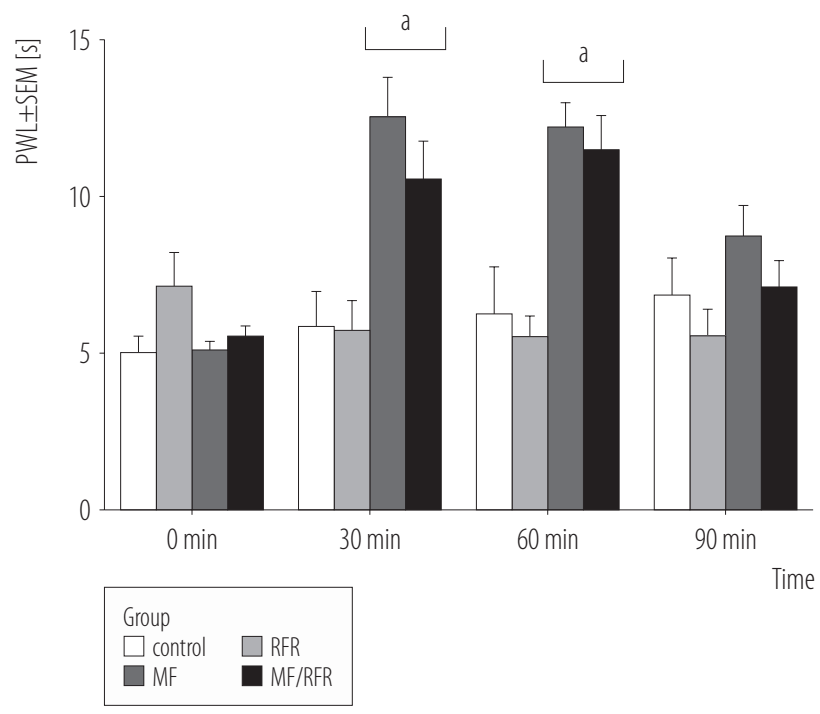

Significant differences from the baseline (ANOVA): ${ }^{\mathrm{a}} \mathrm{p}<0.0005$. $\mathrm{PWL}$ values given as $\mathrm{M} \pm \mathrm{SEM}$.

Figure 2. Paw withdrawal latency (PWL) to a thermal stimulus in healthy rats in 4 groups at the baseline time $(0 \mathrm{~min})$, and at 30, 60 and 90 min after morphine (MF) injection, with a single electromagnetic field exposure (radio-frequency radiation - RFR) or together with morphine (MF/RFR) the study conducted at Military Institute of Hygiene and Epidemiology in Warsaw in 2017

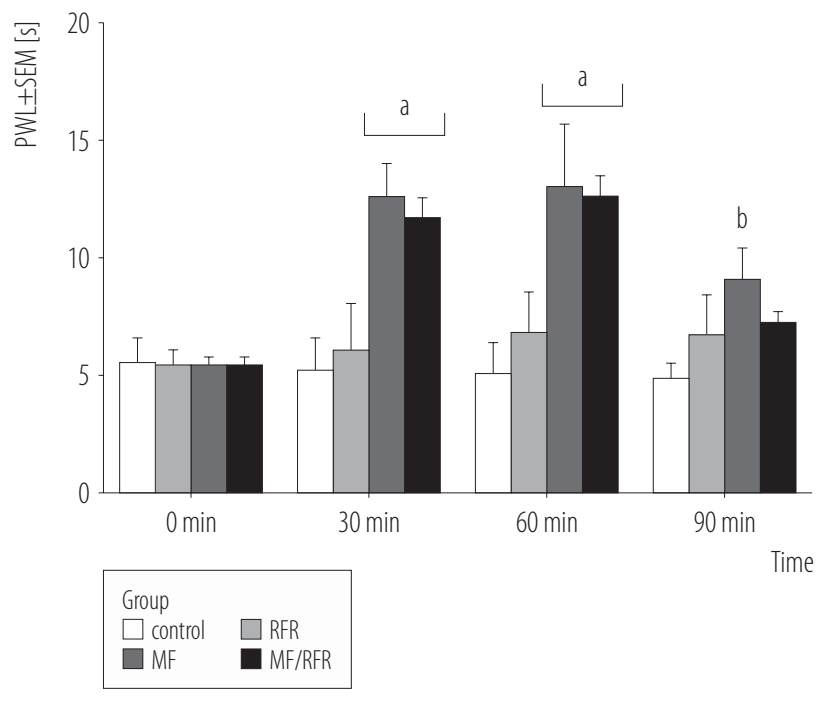

Significant differences from the baseline (ANOVA): ${ }^{\mathrm{a}} \mathrm{p}<0.0005$; ${ }^{\mathrm{b}} \mathrm{p}<0.005$.

$\mathrm{PWL}$ values given as $\mathrm{M} \pm \mathrm{SEM}$.

Figure 3. Paw withdrawal latency (PWL) to a thermal stimulus in healthy rats in 4 groups at the baseline ( $0 \mathrm{~min})$, and at 30,60 and $90 \mathrm{~min}$ after morphine (MF) injection in the dose of $8 \mathrm{mg} / \mathrm{kg}$, with a repeated radio-frequency radiation (RFR) exposure, both alone (RFR) or together with morphine (MF/RFR) - the study conducted at Military Institute of Hygiene and Epidemiology in Warsaw in 2017

the control group, was stable in the repeated RFR-exposure group. However, in the single electromagnetic field exposure group, a gentle increase could be observed even though there was no significant statistical difference in both cases. Interestingly, in the group of rats exposed to RFR without morphine injection, the PWL dropped in single exposure but rose in repeated exposure.

A significant statistical difference, comparing to the control group, was observed in the rats with MF injection and the rats exposed to RFR stimulation along with morphine injection. The antinociceptive effect of MF reached its peak after 30 min of RFR stimulation in the single RFR-exposure group, and after $60 \mathrm{~min}$ in the repeated RFR-exposure group. What is more, after single and repeated exposure, a reduction of PWL after $90 \mathrm{~min}$ could be observed in both groups. 


\section{Rats with inflammation}

The complete Freund's adjuvant injection produced a significant reduction of PWL in each measurement $(30,60$ and $90 \mathrm{~min}$ ) (Figures 4 and 5), but it was not affected by single RFR exposure in the baseline time. The PWL time of inflamed rats after RFR stimulation fluctuated after each measurement. Morphine application induced significant antinociceptive effects at 30 and $60 \mathrm{~min}$ after drug injection in the single stimulation population. Radio-frequency radiation exposure markedly influenced PWL of MF-treated and exposed rats (CFA/MF/RFR), as compared to the baseline in single exposed rats in 30 and 60 min measurement. In addition, electromagnetic field stimulation increased the antinociceptive effects of MF

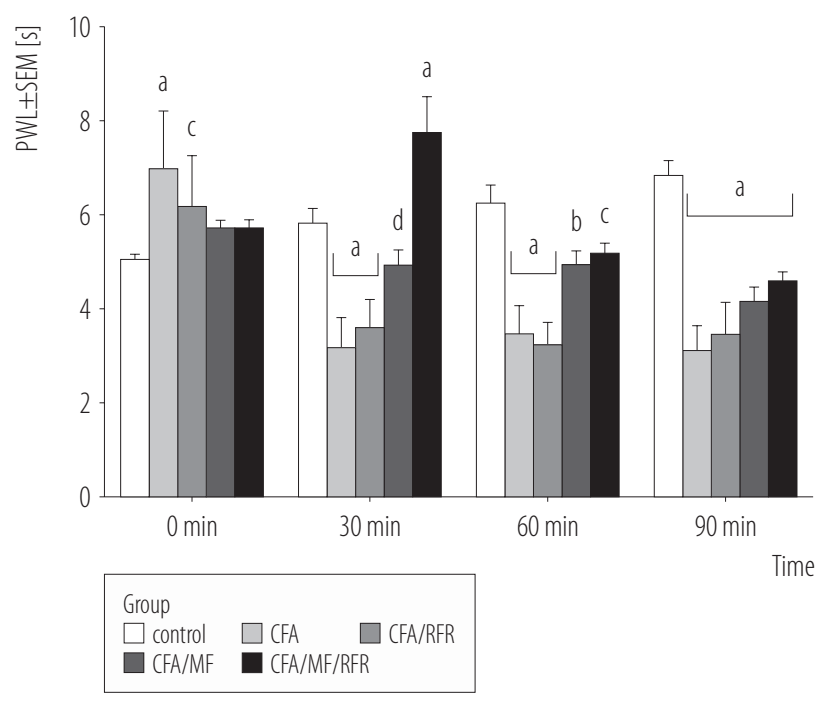

CFA - complete Freund's adjuvant.

Significant differences from the baseline (ANOVA): ${ }^{\mathrm{a}} \mathrm{p}<0.0005$;

${ }^{\mathrm{b}} \mathrm{p}<0.005,{ }^{\mathrm{c}} \mathrm{p}<0.001,{ }^{\mathrm{d}} \mathrm{p}<0.05$.

$\mathrm{PWL}$ values given as means $\mathrm{M} \pm \mathrm{SEM}$.

Figure 4. Paw withdrawal latency (PWL) to a thermal stimulus in rats with inflammation in 4 groups at the baseline time (0 min), and at 30, 60 and $90 \mathrm{~min}$ after morphine (MF) or vehicle injection (CFA - complete Freund's adjuvant), with a single electromagnetic field exposure (radio-frequency radiation RFR; CFA/RFR) or together with morphine (CFA/MF and CFA/MF/RFR) - the study conducted at Military Institute of Hygiene and Epidemiology in Warsaw in 2017

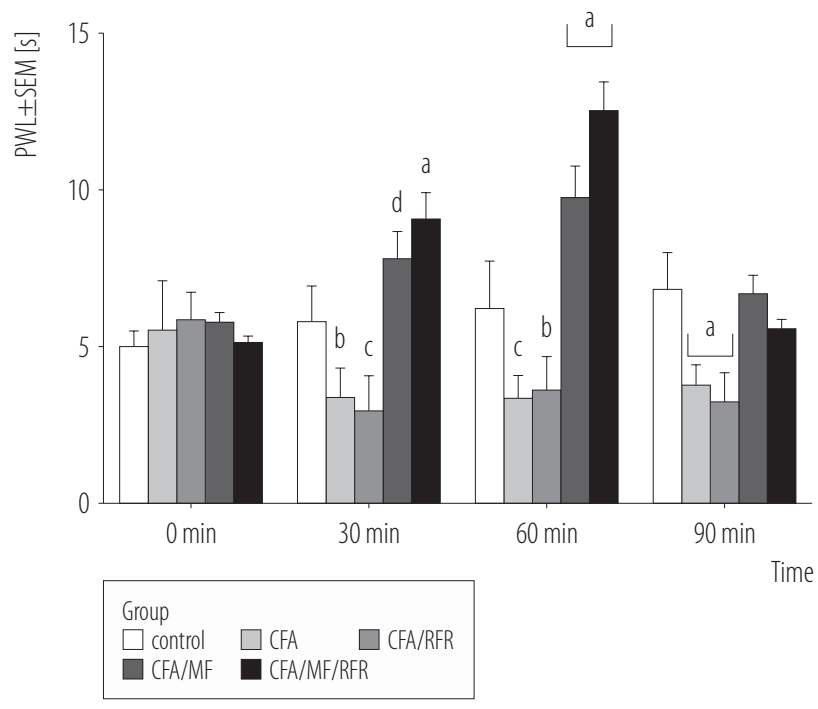

Significant differences from the baseline (ANOVA): ${ }^{\mathrm{a}} \mathrm{p}<0.0005$, ${ }^{\mathrm{b}} \mathrm{p}<0.005,{ }^{\mathrm{c}} \mathrm{p}<0.001,{ }^{\mathrm{d}} \mathrm{p}<0.05$.

$\mathrm{PWL}$ values given as $\mathrm{M} \pm \mathrm{SEM}$.

Figure 5. Paw withdrawal latency (PWL) to a thermal stimulus in rats with inflammation in 4 groups at the baseline time (0 min), and at 30, 60 and 90 min after morphine (MF) or vehicle injection (CFA - complete Freund's adjuvant), with a repeated electromagnetic field (radio-frequency radiation - RFR; CFA/RFR) exposure or together with morphine (CFA/MF and CFA/MF/RFR) - the study conducted at Military Institute of Hygiene and Epidemiology in Warsaw in 2017

after both of these measurements. The PWL recorded was significantly higher than the baseline in the inflammation group (CFA/MF/RFR) after exposure at $30 \mathrm{~min}$ in the repeated exposure group, despite the fact that the PWL time had a downward tendency over time in the repeated CFA/ $\mathrm{MF} / \mathrm{RFR}$ group. These results suggest that both a single and repeated RFR exposure may potentiate the antinociceptive effects of morphine in the inflammation group.

\section{DISCUSSION}

Nociceptive responses and pain sensitivity induced by RFR exposure have been demonstrated in a variety of studies by a number of different investigators. Morphine and a variety of exogenous opiates were used to induce 
analgesia in several studies which investigated the effects of parallel exposure to RFR and opioid administration. The $\mu$-opioid receptors (MOR), widely distributed in the central nervous system, including the spinal cord, are predominantly involved in mediating the analgesic effects of morphine [17].

Morphine and other centrally acting $\mu$-opioid analgesics may be limited in their use in pain treatment, due to several adverse effects, such as the inhibition of gastrointestinal motility, respiratory depression, and tolerance, which may occur apart from their antinociceptive effects [18,19]. The prototypic opiate antagonist, naloxone was used in a number of in vivo studies in order to compare the inhibitory effects of RFR on analgesia. The collected results have shown that naloxone and RFR exposure have similar inhibitory effects on analgesia [20]. Martin et al. [21] obtained contrasting data, which showed that pre-injection with naloxone eliminated the analgesic effects of complex fields. The other functions, in addition to nociception, which are mediated by opioids, such as sensory, emotional, and cognitive functions, may also be influenced by exposure to RFR.

Lei et al. [22] presented that such effects as feeding, spatial learning, locomotor hyperactivity, morphine-induced place preferences, and electrical or pharmacologically induced seizures may be induced by extremely low-frequency (ELF) electromagnetic fields. Therefore, there is a possibility for a broad range of effects of the influence of RFR exposure on opioid-modulated biological functions. However, in recent years several studies have been conducted, which have investigated the effects of electromagnetic fields (RFRs) emitted by mobile phones on cognitive functions, and it is still unclear whether some functions of the central nervous system may be influenced by this environmental factor [23].

There are many examples where RFR radiation by itself has had no observable effect [24], but it may be enhanced or reduced by another agent, such as an opioid drug. Acelli et al. [25] showed that in anesthetized rats pretreated with LPS and exposed for $2 \mathrm{~h}$ to GSM-1800 MHz signals such exposure changed microglial cell morphology and caused significant alterations in neuronal responses to both pure tones and communication sounds.

In this study, it was shown that RFR exposure has an inhibitory effect on pain. Similar results were obtained by Ozdemir et al. [26] who indicated that the application of the extremely low frequency EMF to rats increased the morphine analgesia and reduced morphine analgesic tolerance. These results might be caused by changes in the underlying neurophysiological processes, as suggested by Bailey and Connor [27]. Cytokines are another important factor that may have a significant impact on the RFR and MF effect. Numerous research has shown that electromagnetic field decreases proinflammatory cytokine production. Fairbanks and Wilcox [28] in their research suggested that IL-1, which is a proinflammatory cytokine, was an early signal in the recruitment of endogenous pain facilitatory mechanisms that modulate opioid analgesia. While it can be assumed that tolerance to morphine can be weakened by single RFR stimulation, the same conclusions cannot be drawn for the repeated RFR exposure until the research on the immune response of rats is done. Bearing in mind that a lot of aspects of everyday life may be influenced by RFR exposure, the broad knowledge from the life sciences and medicine should be engaged in explaining possible mechanisms of action.

\section{CONCLUSIONS}

Results of this study show that RFR exposure (1800 MHz) may strengthen the antinociceptive effect of morphine after repeated exposure (5 times for $15 \mathrm{~min}$ on consecutive days) in the group of rats with inflammation. Nevertheless, these findings suggest that electromagnetic exposure at a frequency of $1800 \mathrm{MHz}$ may be a complementary agent for the treatment of chronic pain with opioid drug use. 


\section{REFERENCES}

1. Sirav B, Seyhan N. Blood-brain barrier disruption by continuous-wave radio frequency radiation. Electromagn Biol Med. 2009;28(2):215-22, https://doi.org/10.1080/1536837080 2608738.

2. Sirav B, Seyhan N. Effects of radiofrequency radiation exposure on blood-brain barrier permeability in male and female rats. Electromagn Biol Med. 2011;30(4):253-60.

3. Sirav B, Seyhan N. Effects of GSM modulated radio-frequency electromagnetic radiation on permeability of blood-brain barrier in male and female rats. J Chem Neur. 2016;75(Pt B): 123-7.

4. Bodera P, Stankiewicz W, Antkowiak B, Paluch M, Kieliszek J, Sobiech J, et al. Influence of electromagnetic field (1800 MHz) on lipid peroxidation in brain, blood, liver and kidney in rats. Int J Occup Med Environ Health. 2015;28(4):751-9, https:// doi.org/10.13075/ijomeh.1896.00255.

5. Zecca L, Mantegazza C, Margonato V, Cerretelli P, Caniatti M, Piva F, et al. Biological effects of prolonged exposure to ELF electromagnetic fields in rats: III. $50 \mathrm{~Hz}$ electromagnetic fields. Bioelectromagnetics. 1998;19(1):57-66, https:// doi.org/10.1002/(SICI)1521-186X(1998)19:1<57::AIDBEM7>3.0.CO;2-3.

6. Bao X, Shi Y, Huo X, Song T. A possible involvement of beta-endorphin, substance $\mathrm{P}$, and serotonin in rat analgesia induced by extremely low frequency magnetic field. Bioelectromagnetics. 2006;27(6):467-72, https://doi.org/10.1002/bem. 20232.

7. Wang X, Liu Y, Lei Y, Zhou D, Fu Y, Che Y, et al. Extremely low-frequency electromagnetic field exposure during chronic morphine treatment strengthens downregulation of dopamine D2 receptors in rat dorsal hippocampus after morphine withdrawal. Neurosci Lett. 2008;433(3):178-82.

8. Millan MJ, Czlonkowski A, Morris B, Stein C, Arendt R, Huber A, et al. Inflammation of the hind limb as a model of unilateral, localized pain: influence on multiple opioid systems in the spinal cord of the rat. Pain. 1988;35(3):299-312, https:// doi.org/10.1016/0304-3959(88)90140-6.
9. Colpaert FC. Evidence that adjuvant arthritis in the rat is associated with chronic pain. Pain. 1987;28(2):201-22, https:// doi.org/10.1016/0304-3959(87)90117-5.

10. Iadarola MJ, Brady LS, Draisci G, Dubner R. Enhancement of dynorphin gene expression in spinal cord following experimental inflammation: stimulus specificity, behavioral parameters and opioid receptor binding. Pain. 1988;35(3):313-26, https://doi.org/10.1016/0304-3959(88)90141-8.

11. Hargreaves K, Dubner R, Brown F, Flores C, Joris J. A new and sensitive method for measuring thermal nociception in cutaneous hyperalgesia. Pain. 1988;32(1):77-88, https://doi. org/10.1016/0304-3959(88)90026-7.

12. Galbraith JA, Mrosko BJ, Myers RR. A system to measure thermal nociception. J Neurosci Methods. 1993;49(1-2): 63-8, https://doi.org/10.1016/0165-0270(93)90109-5.

13. Mason PA, Walters TJ, Fanton JW, Erwin DN, Gao JH, Roby JW, et al. Database created from magnetic resonance images of a Sprague-Dawley rat, rhesus monkey, and pigmy goat. FASEB J. 1995;9(5):434-40, https://doi.org/10.1096/ fasebj.9.5.7896016.

14. Roach WP. Radio Frequency Radiation Dosimetry Handbook. 5th ed. Air Force Research Laboratory, Brooks CityBase; 2009.

15. Keenan MA, Stabin MG, Segars WP, Fernald MJ. RADAR realistic animal model series for dose assessment. J Nucl Med. 2010;51(3):471-6.

16. Directive 2010/63/EU of the European Parliament and of the Council of 22 September 2010 on the protection of animals used for scientific purposes Text with EEA relevance. Off J Eur Union L 276/33 (Oct 20, 2010).

17. National Institutes of Health, Office of Animal Care and Use [Internet]. 2009 Guidelines for preparing USDA annual reports and assigning USDA pain and distress categories [cited 2018 Sep 10]. Available from: http://oacu.od.nih.gov/ arac/documents/usda_reports.pdf.

18. Hashimoto T, Saito Y, Yamada K, Hara N, Kirihara Y, Tsuchiya M. Enhancement of morphine analgesic effect with induction of mu-opioid receptor endocytosis in rats. Ane- 
sthesiology. 2006;105(3):574-80, https://doi.org/10.1097/000 00542-200609000-00023.

19. Bonnet MP, Mignon A, Mazoit JX, Ozier Y, Marret E. Analgesic efficacy and adverse effects of epidural morphine compared to parenteral opioids after elective caesarean section: a systematic review. Eur J Pain. 2010;14(9):894.e1-9.

20. Schiller PW. Opioid peptide-derived analgesics. AAPS J. 2005;7(3):560-5.

21. Jeong JH, Choi KB, Yi BC, Chun CH, Sung KY, Sung JY, et al. Effects of extremely low frequency magnetic fields on pain thresholds in mice: roles of melatonin and opioids. J Auton Pharmacol. 2000;20(4):259-64, https://doi. org/10.1046/j.1365-2680.2000.00189.x.

22. Martin LJ, Koren SA, Persinger MA. Thermal analgesic effects from weak, complex magnetic fields and pharmacological interactions. Pharmacol Biochem Behav. 2004;78(2):21727, https://doi.org/10.1016/j.pbb.2004.03.016.

23. Lei Y, Liu T, Wilson FA, Zhou D, Ma Y, Hu X. Effects of extremely low-frequency electromagnetic fields on morphine-induced conditioned place preferences in rats. Neurosci Lett. 2005;390(2):72-5, https://doi.org/10.1016/j.neulet. 2005.08.001.

24. Barth A, Winker R, Ponocny-Seliger E, Mayrhofer W, Ponocny I, Sauter C, et al. A meta-analysis for neurobehavioural effects due to electromagnetic field exposure emitted by
GSM mobile phones. Occup Environ Med. 2008;65(5):3426, https://doi.org/10.1136/oem.2006.031450.

25. Keleş AI ,Ylldırım M, Gedikli Ö, Çolakoğlu S, Kaya H, Bas $\mathrm{O}$, et al. The effects of a continuous 1-h a day $900-\mathrm{MHz}$ electromagnetic field applied throughout early and mid-adolescence on hippocampus morphology and learning behavior in late adolescent male rats. J Chem Neuroanat. 2018;94: 46-53, https://doi.org/10.1016/j.jchemneu.2018.08.006.

26. Occelli F, Lameth J, Adenis V, Huetz C, Lévêque P, Jay TM, et al. A Single Exposure to GSM-1800 MHz Signals in the Course of an Acute Neuroinflammatory Reaction can Alter Neuronal Responses and Microglial Morphology in the Rat Primary Auditory Cortex. Neuroscience. 2018;385: 11-24, https://doi.org/10.1016/j.neuroscience.2018.06.002.

27. Ozdemir E, Demirkazik A, Gursoy S, Taskıran AS, Kilinc O, Arslan G. Effects of extremely low frequency electromagnetic fields on morphine analgesia and tolerance in rats. Gen Physiol Biophys. 2017;36(4):415-22, https://doi.org/10.4149/ gpb_2017008.

28. Bailey CP, Connor M. Opioids: cellular mechanisms of tolerance and physical dependence. Curr Opin Pharmacol. 2005;5:1-9, https://doi.org/10.1016/j.coph.2004.08.012.

29. Fairbanks CA, Wilcox GL. Spinal plasticity of acute opioid tolerance. J Biomed Sci. 2000;7:200-12, https://doi. org/10.1007/BF02255467.

This work is available in Open Access model and licensed under a Creative Commons Attribution-NonCommercial 3.0 Poland License - http://creativecommons.org/ licenses/by-nc/3.0/pl/deed.en. 